\title{
Overall properties of hot, massive stars in the X-ray domain
}

\author{
Yael Nazé \\ FNRS-ULg, Dept AGO, Allée du 6 Août 17, Bât B5C, B4000-Liège, Belgium, \\ email: naze@astro.ulg.ac.be
}

\begin{abstract}
Despite the absence of large surveys, the recent X-ray observatories provide X-ray data for hundreds of massive stars (294 OB stars detected in the 2XMM catalog, 129 OB stars detected in the Chandra Carina Complex Project). Analyzing medium-resolution spectra led to new results on the relationship between the X-ray luminosity and the bolometric luminosity, as well as on the typical properties (plasma temperature, variability) of these objects.
\end{abstract}

Keywords. X-rays: stars, stars: early-type

\section{Introduction}

The X-ray emission of hot stars was serendipitously discovered in 1978-9 (Harnden et al. 1979, Ku et al. 1979, Seward et al. 1979). A link between the X-ray and the visible/bolometric luminosities was readily suggested (Harnden et al. 1979, Pallavicini et al. 1981). With time, this relation was investigated theoretically as well as observationally.

Theoretical considerations show that Lx "naturally" scales with $\dot{M} / v_{\infty}$ and therefore with the bolometric luminosity IF there is "a delicate balance between X-ray emission and absorption" AND "a special form for the radial distribution of wind shocks" (Owocki \& Cohen 1999). In fact, the X-ray filling factor should vary with radial distance from the star as $r^{-s}$ (with $s=0.25-0.4$, but recent fitting of X-ray lines rather yields $s=0$, Cohen et al. 2006).

In-depth observational studies of that $L_{X}-L_{B O L}$ relation were also performed, with two different approaches. On the one hand, Berghöfer et al. (1997) correlated the Rosat All-Sky Survey (RASS) with the Yale bright star catalog (i.e. an heterogeneous population of massive stars, mixing field and cluster objects). Fixing the interstellar absorption, they used the RASS count rates and hardness ratios to derive absorption-corrected X-ray luminosities. They confirmed that $L_{X}=10^{-7} \times L_{B O L}$ down to $10^{38} \mathrm{erg} \mathrm{s}^{-1}(\sim \mathrm{B} 1-1.5)$, though a large scatter $(0.4 \mathrm{dex})$ existed around the relation. On the other hand, XMMNewton and Chandra took sensitive observations of several clusters (i.e. homogeneous populations). In some cases (e.g. NGC6231 Sana et al. 2006, Carina OB1 Antokhin et al. 2008), a detailed analysis of the X-ray spectra, taking into account the interstellar absorption (fixed) but also the potentially additional wind absorption, could be done. It provided precise X-ray ! luminosities while, in parallel, precise bolometric luminosities were derived from dedicated monitorings. From these studies, the scatter around the $L_{X}-L_{B O L}$ relation appears reduced $(0.1-0.2 \mathrm{dex})$. The difference in scatter could have two causes: (1) the improvement in the knowledge of the physical parameters in the recent studies (stellar multiplicity, spectral types and improved photometry from monitorings, detailed X-ray spectral characteristics) or (2) the difference in data analysis (single/several instrument and reduction software, same/different BC and gas-to-dust ratio) and stellar populations (homogeneous vs heterogeneous populations). 


\section{Hot stars in the $2 X M M$}

The 2XMM survey is not an all-sky survey but a compilation of all archival XMMNewton datasets (Watson et al. 2009). Nevertheless, it permits to combine the characteristics of both approaches, i.e. a detailed fitting and a homogeneous treatment of a large (but heterogeneous) sample of stars. Cross-correlating the Reed catalog (Reed 2003, which takes into account the recent monitorings), 294 OB stars were detected, half of these being bright enough for lightcurve/spectral analyses. The results are (Nazé 2009): $\mathrm{X}$-ray variability appears rare on short timescales but rather common on long timescales; the distribution of the detected O-stars is similar to the overall catalog distribution but there is a lack of detections for late and giant B-stars; the spectral fitting revealed the need for both an additional absorption and a second, hot thermal component (often at $\sim 2 \mathrm{keV}$, which is unexpected!) in O-stars; $\mathrm{O}+\mathrm{OB}$ binaries display X-ray luminosities quite similar to those of sin! gle objects. Finally, the $L_{X} / L_{B O L}$ ratio shows a scatter similar to that found in the RASS: cluster-to-cluster differences therefore probably exist.

\section{Hot stars in the CCCP}

The Chandra Carina Complex Project led to the detection of 129 OB stars, half of them having more than 50 recorded counts (i.e. being fit for at least a rough spectral analysis). Spectral fitting was then performed as for the 2XMM sample, on a large but homogeneous sample of stars. The results (Nazé et al. 2010, submitted) confirm the 2XMM findings, i.e. there is a need for additional absorption and 2nd hard thermal component in O-stars and, apart from HD 93403, X-ray bright wind-wind collisions are rare in most $\mathrm{O}+\mathrm{OB}$ binaries. In addition, there is a smooth transition in the X-ray properties from the late O- to the earliest B-stars; the scatter around the $L_{X}-L_{B O L}$ relation is small, as for NGC6231; and there is a shallow increasing trend (to be confirmed!) of medium-to-soft flux ratio with larger $L_{X} / L_{B O L}$ ratio or $L_{B O L}$. Open questions remain, however, e.g. most sources are soft and not luminous and the few overluminous and hard ones are CWB or MCW candidate! s - but what are the other outliers?

\section{References}

Antokhin, I. I., Rauw, G., Vreux, J.-M., van der Hucht, K. A. et al. 2008, A $\mathscr{\mho} A$, 477, 593

Berghöfer, T. W., Schmitt, J. H. M. M., Danner, R., \& Cassinelli, J. P. 1997, A\&SA, 322, 167

Cohen, D. H., Leutenegger, M. A., Grizzard, K. T., Reed, C. L. et al. 2006, MNRAS, 368, 1905

Harnden, Jr., F. R., Branduardi, G., Gorenstein, P., Grindlay, J. et al. 1979, ApJ (Letters), 234, L51

Ku, W. H.-M. \& Chanan, G. A. 1979, ApJ (Letters), 234, L59

Nazé, Y. 2009, A\&A, 506, 1055

Owocki, S. P. \& Cohen, D. H. 1999, ApJ, 520, 833

Pallavicini, R., Golub, L., Rosner, R., Vaiana, G. S. et al. 1981, ApJ, 248, 279

Reed, B. C. 2003, AJ, 125, 2531

Sana, H., Rauw, G., Nazé, Y., Gosset, E. et al. 2006, MNRAS, 372, 661

Seward, F. D., Forman, W. R., Giacconi, R., Griffiths, R. E. et al. 1979, ApJ (Letters), 234, L55

Watson, M. G., Schröder, A. C., Fyfe, D., Page, C. G. et al. 2009, A\&̊A, 493, 339 